\title{
A bijection between noncrossing and nonnesting partitions of types $A$ and $B$
}

\author{
Ricardo Mamede $\|^{1}$ \\ Department of Mathematics, University of Coimbra, \\ 3001-454 Coimbra, Portugal
}

\begin{abstract}
The total number of noncrossing partitions of type $\Psi$ is the $n$th Catalan number $\frac{1}{n+1}\left(\begin{array}{c}2 n \\ n\end{array}\right)$ when $\Psi=A_{n-1}$, and the binomial coefficient $\left(\begin{array}{c}2 n \\ n\end{array}\right)$ when $\Psi=B_{n}$, and these numbers coincide with the correspondent number of nonnesting partitions. For type $A$, there are several bijective proofs of this equality; in particular, the intuitive map, which locally converts each crossing to a nesting, is one of them. In this paper we present a bijection between nonnesting and noncrossing partitions of types $A$ and $B$ that generalizes the type $A$ bijection that locally converts each crossing to a nesting.

Résumé. Le nombre total des partitions non-croisées du type $\Psi$ est le $n$-ème nombre de Catalan $\frac{1}{n+1}\left(\begin{array}{c}2 n \\ n\end{array}\right)$ si $\Psi=$ $A_{n-1}$, et le coefficient binomial $\left(\begin{array}{c}2 n \\ n\end{array}\right)$ si $\Psi=B_{n}$, et ces nombres son coïncidents avec le nombre correspondant des partitions non-emboîtées. Pour le type $A$, il y a plusieurs preuves bijectives de cette égalité; en particulier, la intuitive fonction, qui convertit localement chaque croisée en une emboîtée, c'est un d'entre eux. Dans ce papier nous présentons une bijection entre partitions non-croisées et non-emboîtées des types $A$ et $B$ qui généralise la bijection du type $A$ qui localement convertit chaque croisée en une emboîtée.
\end{abstract}

Keywords: Root systems, noncrossing partitions, nonnesting partitions, bijection

\section{Introduction}

The poset of noncrossing partitions can be defined in a uniform way for any finite Coxeter group $W$. More precisely, for $u, w \in W$, let $u \leq w$ if there is a shortest factorization of $w$ as a product of reflections in $W$ having as prefix such a shortest factorization for $u$. This partial order turns $W$ into a graded poset $A b s(W)$ having the identity 1 as its unique minimal element, where the rank of $w$ is the length of the shortest factorization of $w$ into reflections. Let $c$ be a Coxeter element of $W$. Since all Coxeter elements in $W$ are conjugate to each other, the interval $[1, c]$ in $A b s(W)$ is independent, up to isomorphism, of the choice of $c$. We denote this interval by $\mathrm{NC}(\mathrm{W})$ or by $\mathrm{NC}(\Psi)$, where $\Psi$ is the Cartan-Killing type of $W$, and call it the poset of noncrossing partitions of $W$. It is a self-dual, graded lattice which reduces to the classical lattice of noncrossing partitions of the set $[n]=\{1,2, \ldots, n\}$ defined by Kreweras in [9] when $W$ is the symmetric group $\mathfrak{S}_{n}$ (the Coxeter group of type $A_{n-1}$ ), and to its type $B$ analogue defined

\footnotetext{
${ }^{\dagger}$ This work was supported by CMUC - Centro de Matemática da Universidade de Coimbra. 
by Reiner in [10] when $\mathrm{W}$ is the hyperoctahedral group. The elements in $\mathrm{NC}(\mathrm{W})$ are counted by the generalized Catalan numbers,

$$
\operatorname{Cat}(W)=\prod_{i=1}^{k} \frac{d_{i}+h}{d_{i}},
$$

where $k$ is the number of simple reflections in $W, h$ is the Coxeter number and $d_{1}, \ldots, d_{k}$ are the degrees of the fundamental invariants of $W$ (see [1, 6, 7, 10] for details on the theory of Coxeter groups and noncrossing partitions). When $W$ is the symmetric group $\mathfrak{S}_{n}$, the number $\operatorname{Cat}\left(\mathfrak{S}_{n}\right)$ is just the usual $n$th Catalan number $\frac{1}{n+1}\left(\begin{array}{c}2 n \\ n\end{array}\right)$, and in type $B_{n}$ this number is the binomial coefficient $\left(\begin{array}{c}2 n \\ n\end{array}\right)$.

Nonnesting partitions were defined by Postnikov (see [10, Remark 2]) in a uniform way for all irreducible root systems associated with Weyl groups. If $\Phi$ is such a system, $\Phi^{+}$is a choice of positive roots, and $\Delta$ is the simple system in $\Phi^{+}$, define the root order on $\Phi^{+}$by $\alpha \leq \beta$ if $\alpha, \beta \in \Phi^{+}$and $\beta-\alpha$ is in the positive integer span of the simple roots in $\Delta$. Equipped with this partial order, $\left(\Phi^{+}, \leq\right)$is the root poset of the associated Weyl group $W$. A nonnesting partition on $\Phi$ is just an antichain in root poset $\left(\Phi^{+}, \leq\right)$. Denote by $\mathrm{NN}(\mathrm{W})$ or by $\mathrm{NN}(\Psi)$, where $\Psi$ is the Cartan-Killing type of $W$, the set of all nonnesting partitions of $W$. Postnikov showed that the nonnesting partitions in $\mathrm{NN}(\mathrm{W})$ are also counted by the generalized Catalan number $\operatorname{Cat}(W)$.

In the case of the root systems of type $A$, different bijective proofs of the equality between the cardinals $\left|\mathrm{NN}\left(\mathrm{A}_{\mathrm{n}-1}\right)\right|=\left|\mathrm{NC}\left(\mathrm{A}_{\mathrm{n}-1}\right)\right|$ are known (see [1, 2, 3, 8, 11]). Recently, Christian Stump [11] described a bijection between nonnesting and noncrossing partitions for type $B$, and simultaneously with our work, Alex Fink and Benjamin Giraldo [5] presented a different bijection for each classical group. Our contribution in this paper is to present a uniform proof that $|\mathrm{NN}(\Psi)|=|\mathrm{NC}(\Psi)|$, for $\Psi=A_{n-1}$ and $\Psi=B_{n}$, that generalizes the bijection presented by Armstrong in [1]. All three bijections are distinct, and preserves different statistics. While our bijection preserves the triples $(o p, c l, t r)$ formed by the openers, closers and transients (see the definitions below) of the partitions, and therefore also the number of blocks, the one by Alex Fink and Benjamin Giraldo preserves the type of the partitions but not the triples $(o p, c l, t r)$, and Stump's bijection does not preserve neither the type nor the triples $(o p, c l, t r)$.

\section{Noncrossing and nonnesting partitions of types $A$ and $B$}

A partition of the set $[n]$ is a collection of nonempty disjoint subsets of $[n]$, called blocks, whose union is $[n]$. The type of a partition $\pi$ of $[n]$ is the integer partition formed by the cardinals of the blocks of $\pi$. Let $B$ be a block of $\pi$. Then, the least element of $B$ is called an opener, the greatest element of $B$ is said to be a closer, and the remaining elements of $B$ are called transients. The sets of openers, closers and transients of $\pi$ will be denoted by $o p(\pi), \operatorname{cl}(\pi)$, and $\operatorname{tr}(\pi)$, respectively. The triples $(o p(\pi), \operatorname{tr}(\pi), \operatorname{cl}(\pi))$ encodes useful information about the partition $\pi$. For instance, the number of blocks is $|o p(\pi)|=|c l(\pi)|$, and the number of blocks having only one element is $|o p(\pi) \cap c l(\pi)|$. A partition can be graphically represented by placing the integers $1,2, \ldots, n$ along a line and drawing arcs above the line between $i$ and $j$ whenever $i$ and $j$ lie in the same block and no other element between them does so.

A noncrossing partition of the set $[n]$ is a partition of $[n]$ such that there are no $a<b<c<d$, with $a, c$ belonging to some block of the partition and $b, d$ belonging to some other block. The set of noncrossing partitions of $[n]$, denoted by $\mathrm{NC}(n)$, is a lattice for the refinement order. A nonnesting partition of the set $[n]$ is a partition of $[n]$ such that if $a<b<c<d$ and $a, d$ are consecutive elements of a block, then $b$ and $c$ are not both contained in some other block. The set of nonnesting partitions of $[n]$ will be denoted by 
$\mathrm{NN}(n)$. Graphically, the noncrossing condition means that no two of the arcs cross, while the nonnesting condition means that no two arcs are nested one within the other. For instance, the noncrossing partition $\{\{2,3\},\{1,4,5\}\}$ and the nonnesting partition $\{\{1,3\},\{2,4,5\}\}$ are represented by

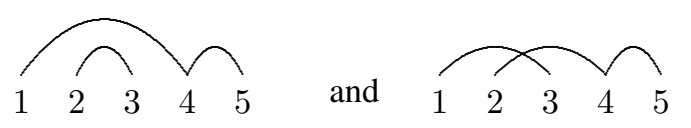

respectively. Both partitions have $\{1,2\}$ as set of openers, $\{3,5\}$ as set of closers and 4 is the only transient. As pointed out in [1], the map that locally converts each crossing to a nesting

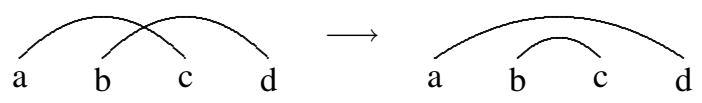

defines a bijection from $\mathrm{NN}(n)$ to $\mathrm{NC}(n)$ that preserves the number of blocks. We will refer to this bijection as the L-map.

We will now review the usual combinatorial realizations of the Coxeter groups of types $A$ and $B$, referring to [7] for any undefined terminology. The Coxeter group $W$ of type $A_{n-1}$ is realized combinatorially as the symmetric group $\mathfrak{S}_{n}$. The permutations in $\mathfrak{S}_{n}$ will be written in cycle notation. The simple generators of $\mathfrak{S}_{n}$ are the transpositions of adjacent integers $(i i+1)$, for $i=1, \ldots, n-1$, and the reflections are the transpositions $(i j)$ for $1 \leq i<j \leq n$. To any permutation $\pi \in \mathfrak{S}_{n}$ we associate the partition of the set $[n]$ given by its cycle structure. This defines a isomorphism between the posets $\mathrm{NC}\left(\mathfrak{S}_{n}\right)$ of noncrossing partitions of $\mathfrak{S}_{n}$, defined in the introduction, and $\mathrm{NC}(n)$, with respect to the Coxeter element $c=(12 \cdots n)$ [4, Theorem 1].

Denoting by $e_{1}, \ldots, e_{n}$ the standard basis of $\mathbb{R}^{n}$, the root system of type $A_{n-1}$ consists of the set of vectors

$$
\Phi=\left\{e_{i}-e_{j}: i \neq j, 1 \leq i, j \leq n\right\}
$$

each root $e_{i}-e_{j}$ corresponding to the transposition $(i j)$. Take

$$
\Phi^{+}=\left\{e_{i}-e_{j} \in \Phi: i>j\right\}
$$

for the set of positive roots and, defining $r_{i}:=e_{i+1}-e_{i}, i=1, \ldots, n-1$, we obtain the simple system $\Delta=\left\{r_{1}, \ldots, r_{n-1}\right\}$ for $\mathfrak{S}_{n}$. Note that

$$
e_{i}-e_{j}=\sum_{k=j}^{i-1} r_{k}, \quad \text { if } i>j
$$

The correspondence between the antichains in the root poset $\left(\Phi^{+}, \leq\right)$and the set of nonnesting partitions of $[n]$ is given by the bijection which sends the positive root $e_{i}-e_{j}$ to the set partition of $[n]$ having 
an arc between vertices $i$ and $j$. For instance, consider the root poset $\left(\Phi^{+}, \leq\right)$of type $A_{4}$ :

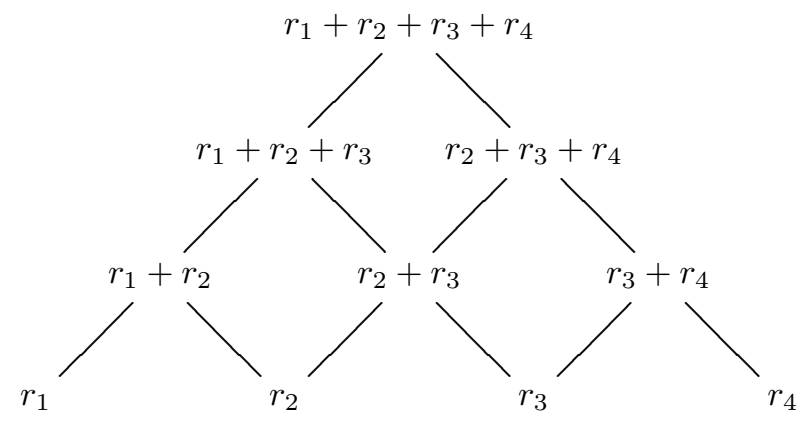

The antichain $r_{1}+r_{2}=e_{3}-e_{1}$ corresponds to the transposition (13) in the symmetric group $\mathfrak{S}_{5}$, and thus to the nonnesting set partition $\{\{1,3\},\{2\},\{4\},\{5\}\}$, while the antichain $\left\{r_{1}+r_{2}, r_{2}+r_{3}, r_{4}\right\}$ corresponds to the product of transpositions $(13)(24)(45)=(13)(245)$ in $\mathfrak{S}_{5}$, and thus to the nonnesting set partition $\{\{1,3\},\{2,4,5\}\}$.

Given a positive root $\alpha=r_{i}+r_{i+1}+\cdots+r_{j} \in \Phi^{+}$, define the support of $\alpha$ as the set $\operatorname{supp}(\alpha)=$ $\left\{r_{i}, r_{i+1}, \ldots, r_{j}\right\}$. The integers $i$ and $j$ will be called, respectively, the first and last indices of $\alpha$, and the roots $r_{i}$ and $r_{j}$ the first and last elements of $\alpha$, respectively. We have the following lemma.

Lemma 2.1 Let $\alpha_{1}, \alpha_{2}$ be two roots in $\Phi^{+}$with first and last indices $i_{1}, j_{1}$ and $i_{2}, j_{2}$, respectively. Then, $\alpha_{1}, \alpha_{2}$ form an antichain if and only if $i_{1}<i_{2}$ and $j_{1}<j_{2}$.

Consider now the Coxeter group $W$ of type $B_{n}$, with its usual combinatorial realization as the hyperoctahedral group of signed permutations of

$$
[ \pm n]:=\{ \pm 1, \pm 2, \ldots, \pm n\} .
$$

These are permutations of $[ \pm n]$ which commute with the involution $i \mapsto-i$. We will write the elements of $W$ in cycle notation, using commas between elements. The simple generators of $W$ are the transposition $(-1,1)$ and the pairs $(-i-1,-i)(i, i+1)$ for $i=1, \ldots, n-1$. The reflections in $W$ are the transpositions $(-i, i)$, for $i=1, \ldots, n$, and the pairs of transpositions $(i, j)(-j,-i)$ for $i \neq j$. Identifying the sets $[ \pm n]$ and $[2 n]$ through the map $i \mapsto i$ for $i \in[n]$ and $i \mapsto n-i$ for $i \in\{-1,-2, \ldots,-n\}$, allows us to identify the hyperoctahedral group $W$ with the subgroup $U$ of $\mathfrak{S}_{2 n}$ which commutes with the permutation $(1, n+1)(2, n+2) \cdots(n, 2 n)$. For example, the signed permutations $(1,3)$ and $(2,-3)(-2,3)$ in the hyperoctahedral group of type $B_{3}$ correspond to the permutations $\left(\begin{array}{ll}1 & 3\end{array}\right)$ and $(26)(53)$ in the symmetric group $\mathfrak{S}_{6}$. It follows that $\mathrm{NC}(U)$ is a sublattice of $\mathrm{NC}\left(\mathfrak{S}_{2 n}\right)$, isomorphic to $\mathrm{NC}(W)$ (see [1]).

The type $B_{n}$ root system consists on the set of $2 n^{2}$ vectors

$$
\Phi=\left\{ \pm e_{i}: 1 \leq i \leq n\right\} \cup\left\{ \pm e_{i} \pm e_{j}: i \neq j, 1 \leq i, j \leq n\right\},
$$

and we take

$$
\Phi^{+}=\left\{e_{i}: 1 \leq i \leq n\right\} \cup\left\{e_{i} \pm e_{j}: 1 \leq j<i \leq n\right\}
$$

as a choice of positive roots. Changing the notation slightly from the one used for $\mathfrak{S}_{n}$, let $r_{1}:=e_{1}$ and $r_{i}:=e_{i}-e_{i-1}$, for $i=2, \ldots, n$. The set

$$
\Delta:=\left\{r_{1}, r_{2}, \ldots, r_{n}\right\}
$$


is a simple system for $W$, and easy computations show that

$$
\begin{gathered}
e_{i}=\sum_{k=1}^{i} r_{k}, \\
e_{i}-e_{j}=\sum_{k=j+1}^{i} r_{k}, \quad \text { if } i>j \\
e_{i}+e_{j}=2 \sum_{k=1}^{j} r_{k}+\sum_{k=j+1}^{i} r_{k}, \quad \text { if } i>j .
\end{gathered}
$$

Each root $e_{i}, e_{i}-e_{j}$ and $e_{i}+e_{j}$ defines a reflection that acts on $\mathbb{R}^{n}$ as the permutation $(i,-i)$, $(i, j)(-i,-j)$ and $(i,-j)(-i, j)$, respectively, and we will identify the roots with the corresponding permutations. For example, consider the root poset of type $B_{3}$ displayed below:

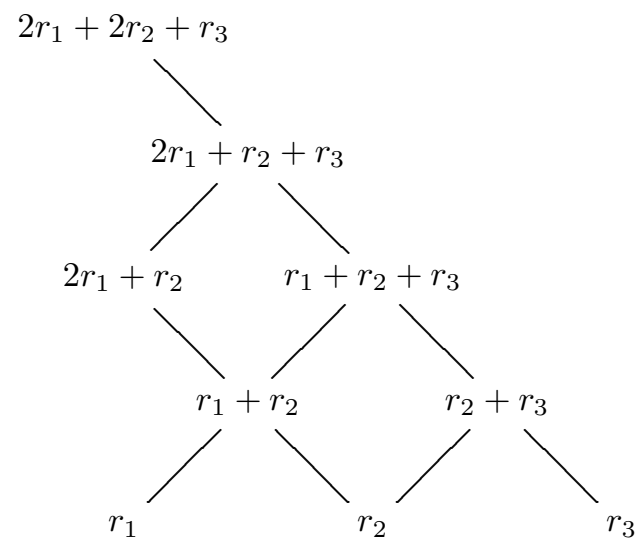

The antichain $\left\{2 r_{1}+r_{2}, r_{2}+r_{3}\right\}$ corresponds to the signed permutation $(1,3,-2)(-1,-3,2)$.

Using the inclusion $W \hookrightarrow \mathfrak{S}_{2 n}$ specified above, we may represent noncrossing and nonnesting partitions of $W$ graphically using the conventions made for its type $A$ analogs. In these representations, we use the integers $-1,-2, \ldots,-n, 1,2, \ldots, n$, or $-n, \ldots,-2,-1,0,1,2, \ldots, n$, respectively for noncrossing and nonnesting partitions, instead of the usual $1,2, \ldots, 2 n$, where the presence of the zero in the ground set for nonnesting partitions is necessary to correctly represent (when present) the arc between a positive number $i$ an its negative (see [2]).

Given a noncrossing or a nonnesting partition $\pi$ of type $B_{n}$, let the set of openers $o p(\pi)$ be formed by the least element of all blocks of $\pi$ having only positive integers; let the set of $\operatorname{closers} c l(\pi)$ be formed by the greatest element of all blocks of $\pi$ having only positive integers and by the absolute values of the least and greatest elements of all blocks having positive and negative integers; and finally let the set of transients $\operatorname{tr}(\pi)$ be formed by all elements of $[n]$ which are not in $o p(\pi) \cup \operatorname{cl}(\pi)$. For instance, if $\pi$ is the nonnesting partition $\{\{-4,4\},\{-1,2\},\{-2,1\},\{3,5\},\{-3,-5\}\}$, represented below, then $o p(\pi)=\{3\}, \operatorname{cl}(\pi)=\{1,2,4,5\}$ and $\operatorname{tr}(\pi)=\emptyset$.

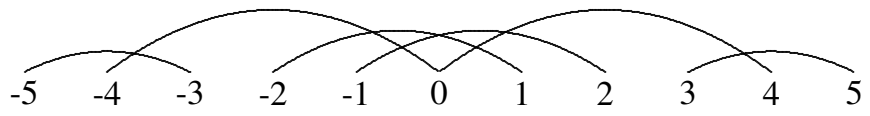


A factor $2 r_{i}$ appearing in a positive root $\alpha$ will be called a double root of $\alpha$. The support of a positive root $\alpha \in \Phi^{+}$is the set of simple and double roots in $\alpha$. Define also the set $\overline{\operatorname{supp}}(\alpha)$ as the set formed by the simple roots appearing in alpha as simple or double roots. For instance, for $\alpha=2 r_{1}+\cdots+2 r_{j}+r_{j+1}+$ $\cdots+r_{i}$ and $\beta=r_{\ell}+\cdots+r_{k}$ we have $\operatorname{supp}(\alpha)=\left\{2 r_{1}, \ldots, 2 r_{j}, r_{j+1}, \ldots, r_{i}\right\}, \overline{\operatorname{supp}}(\alpha)=\left\{r_{1}, \ldots, r_{i}\right\}$, and $\operatorname{supp}(\beta)=\overline{\operatorname{supp}}(\beta)=\left\{\mathrm{r}_{\ell}, \ldots, \mathrm{r}_{\mathrm{k}}\right\}$. The first and last indices of $\alpha$ are, respectively $1, i$ and $\ell, k$. The integer $j$ will be called the last double index in $\alpha$. Define also the set $D_{\alpha}:=\left\{r_{2}, \ldots, r_{j}\right\}$ as the set of simple roots appearing in $\alpha$ as double roots, other than $r_{1}$. We have the following lemma.

Lemma 2.2 Let $\alpha$ and $\beta$ be two roots in $\Phi^{+}$with first and last indices $i, j$ and $i^{\prime}, j^{\prime}$, respectively. If neither $\alpha$ nor $\beta$ have double roots, then $\{\alpha, \beta\}$ is an antichain if and only if $i<i^{\prime}$ and $j<j^{\prime}$. If $\alpha$ has double roots, then $\{\alpha, \beta\}$ is an antichain if and only if $j<j^{\prime}$ and the number of double roots in $\alpha$ is greater than the number of double roots in $\beta$.

\section{Main result}

Let $\Phi$ denote a root system of type $A$ or type $B$, and let $\Phi^{+}$and $\Delta$ be defined as above. In view of lemmas 2.1 and 2.2, we consider antichains $\left\{\alpha_{1}, \ldots, \alpha_{m}\right\}$ in $\Phi^{+}$as ordered $m$-tuples numbered so that if $i_{\ell}$ is the last index of $\alpha_{\ell}$, then $i_{1}<\cdots<i_{m}$.

Definition 1 Given two positive roots $\alpha$ and $\beta$, with $\beta$ having no double roots, and such that the intersection of their supports is nonempty, define their union $\alpha \cup \beta$ and their intersection $\alpha \cap \beta$ as the positive roots with supports

$$
\operatorname{supp}(\alpha \cup \beta):=\operatorname{supp}(\alpha) \cup(\operatorname{supp}(\beta) \backslash \overline{\operatorname{supp}}(\alpha)) \quad \text { and } \quad \operatorname{supp}(\alpha \cap \beta):=\overline{\operatorname{supp}}(\alpha) \cap \operatorname{supp}(\beta) \text {, }
$$

respectively. If moreover $\alpha$ has double roots, then define also their $d$-intersection $\alpha \cap^{d} \beta$ as the positive root with support $\operatorname{supp}\left(\alpha \cap^{\mathrm{d}} \beta\right):=\mathrm{D}_{\alpha} \cap \operatorname{supp}(\beta)$.

Example 2 The union and the intersections of the type $B_{3}$ positive roots $\alpha=2 r_{1}+2 r_{2}+r_{3}$ and $\beta=r_{2}+r_{3}+r_{4}$ are $\alpha \cup \beta=2 r_{1}+2 r_{2}+r_{3}+r_{4}, \alpha \cap^{d} \beta=r_{2}$, and $\alpha \cap \beta=r_{2}+r_{3}$.

An antichain $\left(\alpha_{1}, \ldots, \alpha_{m}\right)$ is said to be connected if the intersection of the supports of any two adjacent roots $\alpha_{i}, \alpha_{i+1}$ is non empty. The connected components

$$
\left(\alpha_{1}, \ldots, \alpha_{i}\right),\left(\alpha_{i+1}, \ldots, \alpha_{j}\right), \ldots,\left(\alpha_{k}, \ldots, \alpha_{m}\right)
$$

of an antichain $\alpha=\left(\alpha_{1}, \ldots, \alpha_{m}\right)$ are the connected sub-antichains of $\alpha$ for which the supports of the union of the roots in any two distinct components are disjoint. For instance, the antichain $\left(r_{1}+r_{2}, r_{2}+\right.$ $\left.r_{3}, r_{4}\right)$ has the connected components $\left(r_{1}+r_{2}, r_{2}+r_{3}\right)$ and $r_{4}$. We will use lower and upper arcs to match two roots in a connected antichain in a geometric manner. Two roots linked by a lower [respectively upper] arc are said to be l-linked [respectively, u-linked]. In what follows we will identify each root with the correspondent permutation.

Definition 3 Define the map $f$ from the set $\mathrm{NN}(\Phi)$ into $\mathrm{NC}(\Phi)$ recursively as follows. When $\alpha_{1}$ is a positive root we set $f\left(\alpha_{1}\right):=\alpha_{1}$. If $\alpha=\left(\alpha_{1}, \ldots, \alpha_{m}\right)$ is a connected antichain with $m>2$, we have two cases: 
(a) If there are no double roots in the antichain, define

$$
f(\alpha):=\left(\bigcup_{k=1}^{m} \alpha_{k}\right) f\left(\bar{\alpha}_{2}, \ldots, \bar{\alpha}_{m}\right),
$$

where $\bar{\alpha}_{k}=\alpha_{k-1} \cap \alpha_{k}$ for $k=2, \ldots, m$.

(b) Assume now that $\alpha_{1}, \ldots, \alpha_{\ell}$ have double roots, for some $\ell \geq 1$, and $\alpha_{\ell+1}, \ldots, \alpha_{m}$ have none. Let $\Gamma_{d}:=\left(\alpha_{1}, \ldots, \alpha_{\ell}\right)$ and $\Gamma:=\left(\alpha_{\ell+1}, \ldots, \alpha_{m}\right)$. We start by introducing l-links as follows.

Let $m^{\prime}$ be the largest index of elements in $\Gamma$ such that the following holds: $\alpha_{m^{\prime}}$ has a first index $i \neq 1$, so that there is a rightmost element, say $\alpha_{k}$, of $\Gamma_{d}$ which has a term $2 r_{i}$. If there is such an integer $m^{\prime}$, l-link $\alpha_{k}$ with $\alpha_{m^{\prime}}$. Then, ignore $\alpha_{k}$ and $\alpha_{m^{\prime}}$ and proceed with the remaining roots as before. This procedure terminates after a finite number of steps (and not all elements of $\alpha$ need to be l-linked).

Next proceed by introducing u-links in $\alpha$. The starting point of u-links, which we consider drawn from right to left, will be elements in $\Gamma$ that have no first index 1 and are not l-linked. We will refer to these elements as admissible roots. So, let $m^{\prime}$ be the smallest integer such that the following holds: $\alpha_{m^{\prime}}$ is an admissible root with first index $i \neq 1$ so that there is a leftmost element, say $\alpha_{k}$ which has $r_{i}$ or $2 r_{i}$ in its support and is not yet $u$-linked to an element on its right. If there is such an integer $m^{\prime}, u$-link $\alpha_{k}$ with $\alpha_{m^{\prime}}$. Remove $\alpha_{m^{\prime}}$ from the set of admissible roots and proceed as before. Again this process terminates after a finite number of steps.

Finally, let $T=\left\{t_{1}<\cdots<t_{p}\right\}$ be the collection of all last double indices of the roots in $\Gamma_{d}$ not l-linked, and all the last indices of the roots in $\alpha$ not $u$-linked to an element on its right. Then, define

$$
f(\alpha):=\pi_{1} \cdots \pi_{\ell} \pi_{0} \theta_{1} \cdots \theta_{q} f\left(\theta_{q+1}, \ldots, \theta_{s}\right),
$$

where for $j=1, \ldots, \ell, \pi_{j}=2 r_{1}+\cdots+2 r_{j^{\prime}}+r_{j^{\prime}+1}+\cdots+r_{j^{\prime \prime}}$, with $j^{\prime}$ and $j^{\prime \prime}$ respectively the leftmost and rightmost integers in $T$ not considered yet; $\pi_{0}$ is either the root $r_{1}+\cdots+r_{i_{j}}$, if the first index of $\alpha_{\ell+1}$ is 1 , with $i_{j}$ the only integer in $T$ not used yet for defining the roots $\pi_{j}$, or the identity otherwise; each $\theta_{j}, j=1, \ldots, q$ is the $d$-intersection of l-linked roots, starting from the rightmost one in $\Gamma_{d}$, and each $\theta_{j}$, $j=q+1, \ldots, s$ is the intersection of $u$-linked roots, starting from the leftmost one in $\Gamma$.

(c) For the general case, if $\left(\alpha_{1}, \ldots, \alpha_{i}\right),\left(\alpha_{i+1}, \ldots, \alpha_{j}\right), \ldots,\left(\alpha_{k}, \ldots, \alpha_{m}\right)$ are the connected components of $\left(\alpha_{1}, \ldots, \alpha_{m}\right)$, let

$$
f\left(\alpha_{1}, \ldots, \alpha_{m}\right):=f\left(\alpha_{1}, \ldots, \alpha_{i}\right) f\left(\alpha_{i+1}, \ldots, \alpha_{j}\right) \cdots f\left(\alpha_{k}, \ldots, \alpha_{m}\right) .
$$

Remark 4 ( $i$ ) Notice that in the type A case, the map $f$ is defined only by conditions $(a)$ and $(c)$ of the above definition. Also, note that if all roots in $\alpha$ have double roots then condition $(b)$ is vacuous and the map $f$ reduces to the identity map. We point out that the number of roots in $f(\alpha)$ is equal to the number of roots in the antichain $\alpha$.

(ii) The sequence $\left(\bar{\alpha}_{2}, \ldots, \bar{\alpha}_{m}\right)$ obtained in step $(a)$ is a (not necessarily connected) antichain. It is easy to check that after all l-links and all u-links are settled, the set $T$ has an odd number of elements if and only if the first index of $\alpha_{\ell+1}$ is 1 . Thus, the root $\pi_{0}$ given in condition $(b)$ is well defined.

(iii) A closer look at the construction of $f$ shows that this map preserves the triples $(o p(\alpha), \operatorname{cl}(\alpha), \operatorname{tr}(\alpha))$ for any antichain $\alpha$. 
We will show that $f$ establishes a bijection between the sets $\mathrm{NN}(\Psi)$ and $\mathrm{NC}(\Psi)$, for $\Psi=A_{n-1}$ or $\Psi=B_{n}$. Before, however, we present some examples.

Example 5 Consider the antichain $\alpha=\left(r_{1}+r_{2}, r_{2}+r_{3}, r_{3}+r_{4}+r_{5}, r_{4}+r_{5}+r_{6}, r_{5}+r_{6}+r_{7}\right)$ in the root poset of type $A_{7}$, corresponding to the permutation $(136)(247)(58)$ in the symmetric group $\mathfrak{S}_{8}$. Applying the map $f$ to $\alpha$, we get the noncrossing partition

$$
\begin{aligned}
f(\alpha) & =\left(r_{1}+\cdots+r_{7}\right) f\left(r_{2}, r_{3}, r_{4}+r_{5}, r_{5}+r_{6}\right) \\
& =\left(r_{1}+\cdots+r_{7}\right) r_{2} r_{3} f\left(r_{4}+r_{5}, r_{5}+r_{6}\right) \\
& =\left(r_{1}+\cdots+r_{7}\right) r_{2} r_{3}\left(r_{4}+r_{5}+r_{6}\right) r_{5} \\
& \equiv(18)(2347)(56),
\end{aligned}
$$

whose graphical representation is given below:

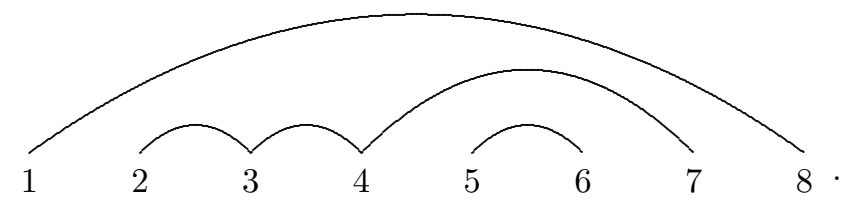

Example 6 Consider now the antichain $\alpha=\left(\alpha_{1}, \alpha_{2}, \alpha_{3}, \alpha_{4}, \alpha_{5}\right)$ in the root poset $B_{9}$, where

$$
\begin{array}{lll}
\alpha_{1}=2 r_{1}+2 r_{2}+2 r_{3}+2 r_{4}+r_{5}, & \alpha_{2}=2 r_{1}+2 r_{2}+r_{3}+r_{4}+r_{5}+r_{6}, \\
\alpha_{3}=r_{1}+r_{2}+r_{3}+r_{4}+r_{5}+r_{6}+r_{7}, & & \alpha_{4}=r_{3}+r_{4}+r_{5}+r_{6}+r_{7}+r_{8}, \\
\alpha_{5}=r_{4}+r_{5}+r_{6}+r_{7}+r_{8}+r_{9} . & &
\end{array}
$$

Following definition 3 we get the l-links and the u-links shown below:

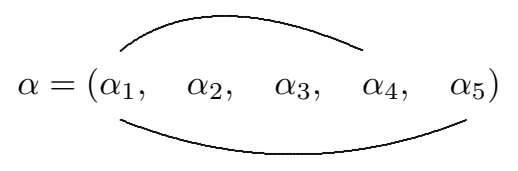

Therefore, $T=\{2,6,7,8,9\}$ and the application of $f$ to $\alpha$ yields:

$$
\begin{aligned}
f(\alpha) & =\left(2 r_{1}+2 r_{2}+r_{3}+\cdots+r_{9}\right)\left(2 r_{1}+\cdots+2 r_{6}+r_{7}+r_{8}\right)\left(r_{1}+\cdots+r_{7}\right) r_{4} f\left(r_{3}+r_{4}+r_{5}\right) \\
& \equiv(2,-9)(-2,9)(6,-8)(-6,8)(7,-7)(3,4)(-3,-4)(2,5)(-2,-5) \\
& =(2,5,-9)(-2,-5,9)(6,-8)(-6,8)(7,-7)(3,4)(-3,-4) .
\end{aligned}
$$

The image $f(\alpha)$ is a noncrossing partition in $[ \pm 9]$, as we may check in its representation:

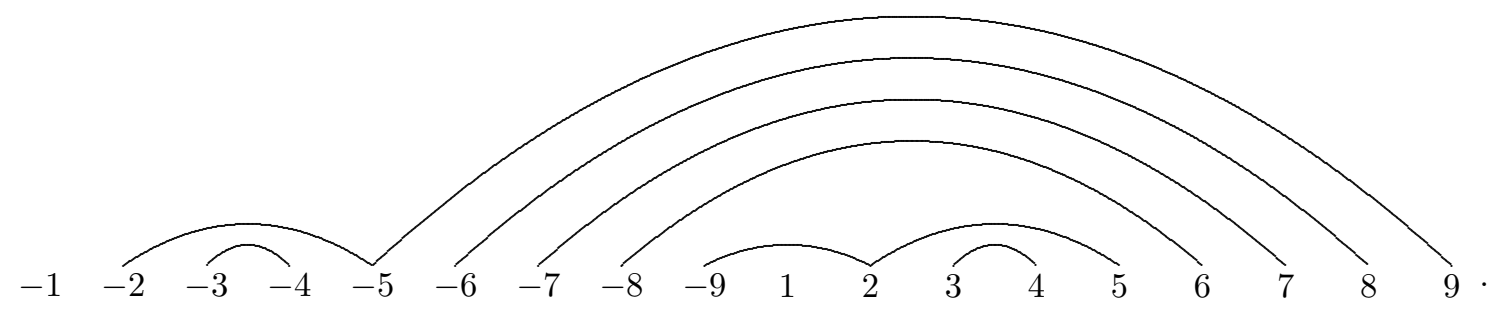


Lemma 3.1 If $\alpha \in \mathrm{NN}\left(B_{n}\right)$ then $f(\alpha) \in \mathrm{NC}\left(B_{n}\right)$.

Proof: Let $\alpha=\left(\alpha_{1}, \ldots, \alpha_{m}\right)$ be an antichain in the root poset of type $B_{n}$, and let $\left(\alpha_{1}, \ldots, \alpha_{w}\right)$ be its first connected component. Start by assuming that none of the positive roots in $\alpha$ have the simple root $r_{1}$ nor the double root $2 r_{1}$. We will use induction on $m \geq 1$ to show that in this case $f(\alpha)$ is a noncrossing partition on the set $\{i-1, \ldots, q,-(i-1), \ldots,-q\}$, where $i$ is the fist index of $\alpha_{1}$ and $q$ is the last index of $\alpha_{m}$, and such that each positive integer is sent to another positive integer. The result is clear when $m=1$. So, let $m \geq 2$ and assume the result for antichains of length less than, or equal to $m-1$. Then, we may write

$$
f(\alpha)=\left(\bigcup_{k=1}^{w} \alpha_{k}\right) f\left(\bar{\alpha}_{2}, \ldots, \bar{\alpha}_{w}\right) f\left(\alpha_{w+1}, \ldots, \alpha_{m}\right),
$$

where each $\bar{\alpha}_{k}=\alpha_{k-1} \cap \alpha_{k}$, for $k=2, \ldots, w$. By the inductive step, $f\left(\bar{\alpha}_{2}, \ldots, \bar{\alpha}_{w}\right) \equiv \pi_{1}$ and $f\left(\alpha_{w+1}, \ldots, \alpha_{m}\right) \equiv \pi_{2}$ are noncrossing partitions on the sets

$$
\{a-1, \ldots, b,-(a-1), \ldots,-b\} \text { and }\{p-1, \ldots, q,-(p-1), \ldots,-q\},
$$

respectively, where $a$ and $p$ are the first indices of $\bar{\alpha}_{2}$ and $\alpha_{w+1}$, respectively, and $b$ and $q$ are the last indices of $\bar{\alpha}_{w}$ and $\alpha_{m}$, respectively. Moreover, all positive integers are sent to positive ones by $\pi_{1}$ and $\pi_{2}$. Denoting by $j$ the last index of $\alpha_{w}$, we get $\bigcup_{k=1}^{w} \alpha_{k}=r_{i}+\cdots+r_{j} \equiv(i-1, j)(-(i-1),-j)$ with $i-1<a-1<b<j \leq p-1<q$. Therefore

$$
f(\alpha) \equiv(i-1, j)(-(i-1),-j) \pi_{1} \pi_{2}
$$

is a noncrossing partition on the set $\{i-1, \ldots, q,-(i-1), \ldots,-q\}$ sending each positive integer to another positive integer.

Note that for the rest of the proof, we may assume without loss of generality that $\alpha$ is connected, since none of the connected components of an antichain, except possible for the first one, have double roots, and therefore their images are noncrossing partitions sending each positive integer to another positive integer.

Suppose now that the first element of $\alpha_{1}$ is $r_{1}$. We will show that $f(\alpha)$ is a noncrossing partition on the set $\{i-1, \ldots, q,-(i-1), \ldots,-q\}$, where $i$ is the first index of $\alpha_{2}$ and $q$ is the last index of $\alpha_{m}$, and such that one and only one positive integer is sent to a negative one. The result is certainly true for $m=1$, and when $m>1$ we have

$$
f(\alpha)=\left(\bigcup_{k=1}^{m} \alpha_{k}\right) f\left(\bar{\alpha}_{2}, \ldots, \bar{\alpha}_{m}\right),
$$

where $\bigcup_{k=1}^{m} \alpha_{k} \equiv(q,-q)$, and $\bar{\alpha}_{k}=\alpha_{k-1} \cap \alpha_{k}$ for $k=2, \ldots, m$. By the previous case, $f\left(\bar{\alpha}_{2}, \ldots, \bar{\alpha}_{m}\right) \equiv$ $\pi$ is a noncrossing partition on the set $\{i-1, \ldots, j,-(i-1), \ldots,-j\}$, with $i$ the first index of $\alpha_{2}$ and $j<q$ the last index of $\alpha_{m-1}$. Therefore, $f(\alpha) \equiv(q,-q) \pi$ is a noncrossing partition satisfying the desired conditions.

Next, assume that $\alpha$ satisfies condition $(b)$ of definition 3 , and consider its image

$$
f(\alpha)=\pi_{1} \cdots \pi_{\ell} \pi_{0} \theta_{1} \cdots \theta_{q} f\left(\theta_{q+1}, \ldots, \theta_{s}\right) .
$$


By the construction of the set $T$, it follows that each $D_{\alpha_{j}}, j=1, \ldots, \ell$, is contained in $D_{\pi_{i}}$, for some $i=1, \ldots, \ell$, and that $\pi_{1} \cdots \pi_{\ell} \pi_{0}$ is a noncrossing partition, sending each nonfixed positive integer to a negative one. Note also that the support of each $\theta_{j}, j=1, \ldots, q$, is contained in some $D_{\alpha_{i}}, i=1, \ldots, \ell$, and therefore, in some $D_{\pi_{i}}, i=1, \ldots, \ell$. Moreover, the supports of any two roots $\theta_{i}$ and $\theta_{j}, 1 \leq i, j \leq q$, are either disjoint, or one of them is contained into the other one. Therefore $\theta_{1} \cdots \theta_{q}$ is a noncrossing partition sending each nonfixed positive integer into another positive integer. By the previous cases, $f\left(\theta_{q+1}, \ldots, \theta_{s}\right)$ is also a noncrossing partition sending each nonfixed positive integer into another positive integer. Again by the construction of the set $T$, we find that the support of each $\theta_{j}, j=q+1, \ldots, s$, is either contained in some $D_{\pi_{i}}$, or it does not intersect $D_{\pi_{\ell}}$. For each $j=1, \ldots, q$ and $i=q+1, \ldots, s$, either we have $\operatorname{supp}\left(\theta_{\mathrm{i}}\right) \cap \operatorname{supp}\left(\theta_{\mathrm{j}}\right)=\emptyset$, or $\operatorname{supp}\left(\theta_{\mathrm{i}}\right) \supseteq \operatorname{supp}\left(\theta_{\mathrm{j}}\right)$, this last case happening when $\theta_{i}$ arises from the intersection of two u-linked roots $\alpha_{u} \in \Gamma_{d}$ and $\alpha_{v} \in \Gamma$, and there is some $\alpha_{v+k} \in \Gamma, k \geq 1$, l-linked to $\alpha_{u}$, whose d-intersection gives $\theta_{j}$. Therefore, it follows that $f(\alpha)$ is noncrossing.

With some minor adaptations, the proof of lemma 3.1, in the case where neither the simple root $r_{1}$ nor the double root $2 r_{1}$ are present in $\alpha$, gives the type $A$ analog of the previous result.

Corollary 3.2 If $\alpha \in \mathrm{NN}\left(A_{n-1}\right)$ then $f(\alpha) \in \mathrm{NC}\left(A_{n-1}\right)$.

We will now construct the inverse function of $f$, thus showing that $f$ establishes a bijection between the sets $\mathrm{NN}(\Psi)$ and $\mathrm{NC}(\Psi)$, for $\Psi=A_{n-1}$ or $\Psi=B_{n}$. For that propose, recall the following property.

Lemma 3.3 Two distinct transpositions $(a, b)$ and $(i, j)$ in $\mathfrak{S}_{n}$ commute if and only if the sets $\{i, j\}$ and $\{a, b\}$ are disjoint.

If $\pi_{1} \cdots \pi_{p}$ is the cycle structure of a signed permutation $\pi$, then for each cycle $\pi_{i}=(i j \cdots k)$ there is another cycle $\pi_{j}=(-i-j \cdots-k)$. Denote by $\pi_{i}^{\prime}$ the cycle in $\left\{\pi_{i}, \pi_{j}\right\}$ having the smallest positive integer (when $\pi_{i}=\pi_{j}$ then $\pi_{i}^{\prime}$ is just $\pi_{i}$ ), and call positive cycle structure to the subword of $\pi_{1} \cdots \pi_{p}$ formed by the cycles $\pi_{i}^{\prime}$. Extend this definition to permutations in $\mathfrak{S}_{n}$ by identifying positive cycle structure with cycle structure.

Theorem 3.4 The map $f$ is a bijection between the sets $\mathrm{NN}(\Psi)$ and $\mathrm{NC}(\Psi)$, for $\Psi=A_{n-1}$ or $\Psi=B_{n}$, which preserves the triples $(o p(\pi), c l(\pi), \operatorname{tr}(\pi))$.

Proof: We will construct the inverse map $g: \mathrm{NC}(\Psi) \rightarrow \mathrm{NN}(\Psi)$ of $f$. Given $\pi \in \mathrm{NC}(\Psi)$, let $\pi_{1} \cdots \pi_{s}$ be its positive cycle structure. Replace each cycle $\pi_{i}=\left(i_{1} i_{2} \cdots i_{k}\right)$ by $\left(i_{1} i_{2}\right)\left(i_{2} i_{3}\right) \cdots\left(i_{k-1} i_{k}\right)$, if $i_{\ell}>0$ for $\ell=1, \ldots, k$, or by

$$
\pi_{i}=\left(i_{1} i_{j+1}\right)\left(i_{1} i_{2}\right)\left(i_{2} i_{3}\right) \cdots\left(i_{j-1} i_{j}\right)\left(i_{j+1} i_{j+2}\right) \cdots\left(i_{k-1} i_{k}\right),
$$

if $i_{\ell}>0$ for $\ell=1, \ldots, j$, and $i_{\ell}<0$ for $\ell=j+1, \ldots, k$. Next, baring in mind lemma 3.3 and recalling that $\pi$ is noncrossing, move all transpositions $(i, j)$, with $i>0$ and $j<0$ (if any), to the leftmost positions and order them by its least positive element, and order all remaining transpositions $(i, j)$, with $i, j>0$, by its least positive integer. Replace each transposition $(i j)$ by its correspondent root in the root system of type $\Psi$, and let

$$
\left(\alpha_{1}, \ldots, \alpha_{k}\right)\left(\alpha_{k+1}, \ldots, \alpha_{\ell}\right) \cdots\left(\alpha_{m}, \ldots, \alpha_{n}\right)
$$

be the correspondent sequence of roots, divided by its connected components. Note that given two distinct roots in (1), the sets formed by the first and last indices, if there are no double roots, or by the last and last double indices, otherwise, are clearly disjoint. 
We start by considering that the sequence (1) has only one connected component $\left(\alpha_{1}, \ldots, \alpha_{k}\right)$. Let $\Gamma_{d}=\left(\alpha_{1}, \ldots, \alpha_{r}\right)$ be the subsequence formed by the roots having double roots, and denote by $\Gamma=$ $\left(\alpha_{r+1}, \ldots, \alpha_{k}\right)$ the remaining subsequence. Define $\Gamma^{\prime}=\Gamma_{d}^{\prime}=\emptyset$. If $\Gamma_{d}$ is not empty and $r \neq k$, apply the following algorithm:

Let $\bar{\Gamma}$ be the subsequence of $\Gamma$ obtained by striking out the root $\alpha_{r+1}$ if its first index is 1 . While $\bar{\Gamma} \neq \emptyset$, repeat the following steps:

(i) Let $\alpha_{i}$ be the leftmost root in $\bar{\Gamma}$ and check if $\operatorname{supp}\left(\alpha_{\mathrm{i}}\right) \subseteq \mathrm{D}_{\alpha_{\mathrm{j}}}$, for some $\alpha_{j} \in \Gamma_{d} \backslash \Gamma_{d}^{\prime}$.

(ii) If so, let $\alpha_{i j}$ be the rightmost root in $\Gamma_{d} \backslash \Gamma_{d}^{\prime}$ with this property. Update $\Gamma^{\prime}$ by including in it the rightmost root $\bar{\alpha}$ of $\bar{\Gamma}$ whose support is contained in $\operatorname{supp}\left(\alpha_{\mathrm{i}}\right)$. Update $\bar{\Gamma}$ by striking out the $\operatorname{root} \bar{\alpha}$ and update $\Gamma_{d}^{\prime}$ by including in this set the root $\alpha_{i_{j}}$.

(iii) Otherwise, update $\bar{\Gamma}$ by striking out the $\operatorname{root} \alpha_{i}$.

Next, let $T=\left\{t_{1}>\cdots>t_{r}\right\}$ be the set formed by all last double indices of the roots in $\Gamma_{d} \backslash \Gamma_{d}^{\prime}$ and by the last indices of the roots in $\Gamma^{\prime}$; let $F_{s t}=\left\{f_{r+1}<\cdots<f_{k}\right\}$ be the set formed by the first indices of the roots in $\Gamma$, and let $L_{s t}=\left\{\ell_{1}<\cdots<\ell_{k}\right\}$ be the set formed by the last indices of the roots in $\left(\Gamma \backslash \Gamma^{\prime}\right) \cup \Gamma_{d}$ and by the last double indices of the roots in $\Gamma_{d}^{\prime}$. By this construction, we have $f_{i}<\ell_{i}$ for $i=1, \ldots, r$, and $f_{i}<\ell_{i}$, for $i=r+1, \ldots, k$. Then, define

$$
g(\pi)=\left(\bar{\alpha}_{1}, \ldots, \bar{\alpha}_{k}\right)
$$

where for $i=1, \ldots, r, \bar{\alpha}_{i}=2 r_{1}+\cdots+2 r_{t_{i}}+r_{t_{i}+1}+\cdots+r_{\ell_{i}}$, and for $i=r+1, \ldots, k, \bar{\alpha}_{i}=r_{f_{i}}+\cdots+r_{\ell_{i}}$.

For the general case define

$$
g(\pi)=g\left(\alpha_{1}, \ldots, \alpha_{k}\right) g\left(\alpha_{k+1}, \ldots, \alpha_{\ell}\right) \cdots g\left(\alpha_{m}, \ldots, \alpha_{n}\right)
$$

It is clear from this construction that $g(\pi)$ is an antichain in the root poset of type $\Psi$. Moreover, a closer look at the construction of the map $f$ shows that $g$ is the inverse of $f$. Thus, $f$ (and $g$ ) establishes a bijection between nonnesting and noncrossing partitions of types $A$ and $B$.

In the following examples we illustrate the application of the map $g$.

Example 7 Consider the cycle structure of the noncrossing partition $\pi=(18)(2347)(56)$ in the symmetric group $\mathfrak{S}_{8}$ used in example 5 . Following the proof of theorem 3.4 write

$$
\begin{aligned}
\pi & \equiv(18)(2347)(56) \\
& =(18)(23)(34)(47)(56) \\
& \equiv\left(r_{1}+\cdots+r_{7}\right) r_{2} r_{3}\left(r_{4}+r_{5}+r_{6}\right) r_{5}
\end{aligned}
$$

Note that $\pi$ has only one connected component, and there are no double roots. Next define the sets

$$
F_{s t}=\{1,2,3,4,5\}, \text { and } L_{s t}=\{2,3,5,6,7\}
$$

Thus, we find that the image of $\pi$ by the map $g$ is the antichain

$$
g(\pi)=\left(r_{1}+r_{2}, r_{2}+r_{3}, r_{3}+r_{4}+r_{5}, r_{4}+r_{5}+r_{6}, r_{5}+r_{6}+r_{7}\right)
$$


Example 8 Consider now the noncrossing partition

$$
\pi=(2,5,-9)(-2,-5,9)(6,-8)(-6,8)(7,-7)(3,4)(-3,-4)
$$

obtained in example 6 Its positive cycle structure is

$$
(2,-9)(2,5)(6,-8)(7,-7)(3,4)=(2,-9)(6,-8)(7,-7)(2,5)(3,4)
$$

and thus we get

$$
\pi \equiv\left(2 r_{1}+2 r_{2}+r_{3}+\cdots+r_{9}, 2 r_{1}+\cdots+2 r_{6}+r_{7}+r_{8}, r_{1}+\cdots+r_{7}, r_{3}+r_{4}+r_{5}, r_{4}\right) .
$$

Next, construct the sets

$$
\begin{aligned}
& T=\{4,2\}, \quad F_{s t}=\{1,3,4\} \\
& L_{s t}=\{5,6,7,8,9\} .
\end{aligned}
$$

Therefore, the image of $\pi$ by the map $g$ is the antichain

$$
\left(2 r_{1}+\cdots+2 r_{4}+r_{5}, 2 r_{1}+2 r_{2}+r_{3}+\cdots+r_{6}, r_{1}+\cdots+r_{7}, r_{3}+\cdots+r_{8}, r_{4}+\cdots+r_{9}\right) .
$$

Finally, in the next result we prove that the map $f$ generalizes the bijection that locally converts each crossing to a nesting.

Theorem 3.5 When restricted to the type $A_{n-1}$ case, the map $f$ coincides with the L-map.

Proof: Let $\alpha=\left(\alpha_{1}, \ldots, \alpha_{m}\right)$ be an antichain in the root poset of type $A_{n-1}$. The result will be handled by induction over $m \geq 1$. Without loss of generality, we may assume that $\alpha$ is connected, since otherwise there is an integer $1<k<n-1$ such that each integer less (resp. greater) than $k$ is sent by $\alpha$ to an integer that still is less (resp. greater) that $k$. Therefore, the same happens with the image of $\alpha$ by either the map $f$ or the L-map.

The result is vacuous when $m=1$, and when $m=2$, the only connected nonnesting partition which does not stay invariant under the maps $f$ and $L$ is $\alpha=\left(r_{i}+\cdots+r_{i^{\prime}}\right)\left(r_{j}+\cdots+r_{j^{\prime}}\right)$, for some integers $1 \leq i<j<i^{\prime}<j^{\prime} \leq n-1$. In this case, the equality between $f$ and the L-map is obvious. So, let $m>2$ and assume the result for antichains of length $\leq m-1$. Let $i$ and $j$ be, respectively, the first and last indices of $\alpha_{1}$ and $\alpha_{m}$. Then,

$$
f(\alpha)=\left(r_{i}+\cdots+r_{j}\right) f\left(\bar{\alpha}_{2}, \ldots, \bar{\alpha}_{m}\right),
$$

where each $\bar{\alpha}_{k}=\alpha_{k-1} \cap \alpha_{k}$ for $k \geq 2$, and the antichain $\left(\bar{\alpha}_{2}, \ldots, \bar{\alpha}_{m}\right)$ is clearly nonnesting, and not necessarily connected. By the inductive step, $f\left(\bar{\alpha}_{2}, \ldots, \bar{\alpha}_{m}\right)=L\left(\bar{\alpha}_{2}, \ldots, \bar{\alpha}_{m}\right)$. Moreover, note that converting, from left to right, each local crossing between the first root and the leftmost root in $\alpha$ whose arcs cross, into a nesting gives, precisely,

$$
\left(r_{i}+\cdots+r_{j}\right) L\left(\bar{\alpha}_{2}, \ldots, \bar{\alpha}_{m}\right)
$$

and this operation may be considered the first step of the L-map. Thus, we find that $f(\alpha)=L(\alpha)$. 
Example 9 Consider the antichain $\alpha=\left(r_{1}+r_{2}+r_{3}, r_{2}+r_{3}+r_{4}+r_{5}, r_{3}+r_{4}+r_{5}+r_{6}, r_{5}+r_{6}+r_{7}\right)$ in the root poset of type $A_{7}$. Applying the map $f$ we get

$$
\begin{aligned}
f(\alpha) & =\left(r_{1}+\cdots+r_{7}\right) f\left(r_{2}+r_{3}, r_{3}+r_{4}+r_{5}, r_{5}+r_{6}\right) \\
& =\left(r_{1}+\cdots+r_{7}\right)\left(r_{2}+r_{3}+r_{4}+r_{5}+r_{6}\right) f\left(r_{3}, r_{5}\right) \\
& =\left(r_{1}+\cdots+r_{7}\right)\left(r_{2}+r_{3}+r_{4}+r_{5}+r_{6}\right) r_{3} r_{5} \equiv(18)(27)(34)(56) .
\end{aligned}
$$

On the other hand, applying the L-map to each crossing between the first root and the leftmost root in $\alpha$ whose arcs cross, we get successively

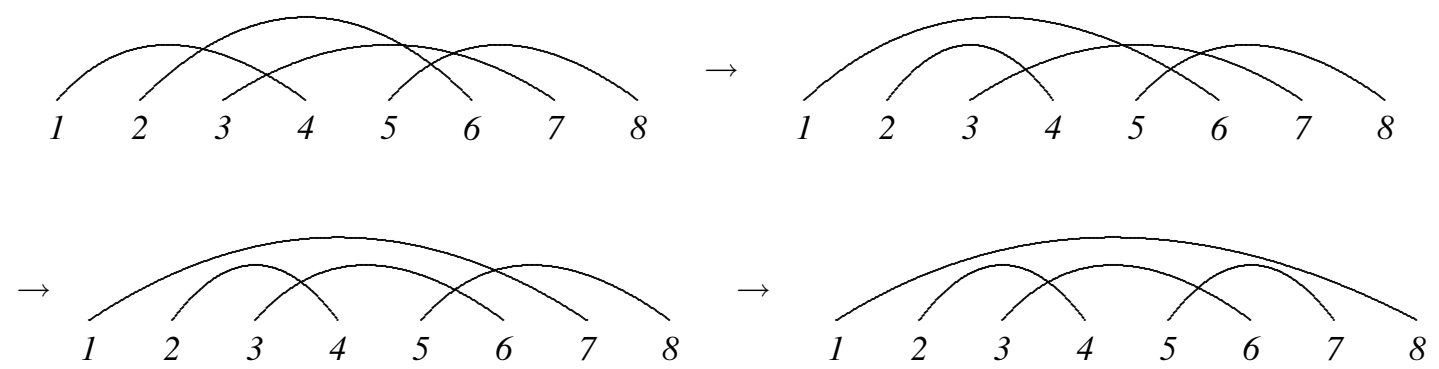

Thus, in the first step of the L-map, we get $L(\alpha)=\left(r_{1}+\cdots+r_{7}\right) L\left(r_{2}+r_{3}, r_{3}+r_{4}+r_{5}, r_{5}+r_{6}\right)$. Continuing the application of the L-map, now replacing, by a nesting, each crossing between the second root and the leftmost root in $\alpha$ whose arcs cross, we get

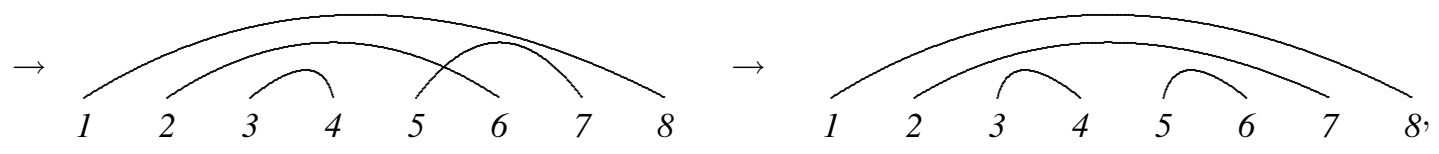

and therefore, we have $L(\alpha)=\left(r_{1}+\cdots+r_{7}\right)\left(r_{2}+r_{3}+r_{4}+r_{5}+r_{6}\right) r_{3} r_{5}=f(\alpha)$.

Acknowledgements. The author thanks Christian Krattenthaler and Alexander Kovacec for useful comments and suggestions.

\section{References}

[1] D. Armstrong, Generalized Noncrossing Partitions and Combinatorics of Coxeter Groups, preprint, arXiv:math/0611106v2 [math.CO].

[2] C.A. Athanasiadis, On noncrossing and nonnesting partitions for classical reflection groups, Elec. J. Comb. 5 (1998), Research Paper 42, 16pp (electronic).

[3] C.A. Athanasiadis and V. Reiner, Noncrossing partitions for the group $D_{n}$, SIAM J. Discrete Math. 18 (2004), 397-417.

[4] P. Biane, Some properties of crossings and partitions, Discrete Math. 175 (1997), 41-53.

[5] A. Fink and B. I. Giraldo, Bijections between noncrossing and nonnesting partitions for classical reflection groups, preprint, arXiv:math/0810.2613v1 [math.CO]. 
[6] L.C. Grove and C.T. Benson, Finite Reflection Groups, Springer-Verlag, New York, 1996.

[7] J. E. Humphreys, Reflection Groups and Coxeter Groups, Cambridge Studies in Advanced Mathematics, vol. 29, Cambridge Univ. Press, Cambridge, 1990.

[8] A. Kasraoui and J. Zeng, Distribution of crossings, nestings and alignments of two edges in matchings and partitions, preprint, arXiv:math/0601081v1 [math.CO].

[9] G. Kreweras, Sur les partitions non-croisées d'un cycle, Discrete Math., 1 (1972), pp. 333-350.

[10] V. Reiner, Non-crossing partitions for classical reflection groups, Discrete Math. 177 (1997), 195222.

[11] C. Stump, Non-crossing partitions, non-nesting partitions and Coxeter sortable elements in types A and $B$, preprint, arXiv:0808.2822v1 [math.CO]. 